\title{
从 $\alpha$-三氟甲基苄溴合成烷基硫酸盐一一脱卤亚磺化反应的延伸
}

\author{
付晓林 ${ }^{a}$ 孙 岩*, 赵志刚*,a 郭 勇 ${ }^{b}$ 陈庆云 ${ }^{b}$ 念保义 ${ }^{c}$ \\ ( ${ }^{a}$ 西南民族大学化学与环境保护工程学院 成都 610041) \\ ${ }^{b}$ 中国科学院上海有机化学研究所 上海 200032) \\ $\left({ }^{c}\right.$ 三明学院三明氟化工产业技术研究院 三明 365004)
}

\begin{abstract}
摘要 脱卤亚磺化反应是引入氟烷基基团的一种常用方法. 探索了 $\alpha$-三氟甲基苄溴在脱卤亚磺化条件下的反应, 发现 产物并不是亚磺酸盐 $\left[\mathrm{ArCH}\left(\mathrm{CF}_{3}\right) \mathrm{SO}_{2} \mathrm{Na}\right.$ ], 而是烷基硫酸盐 $\left[\mathrm{ArCH}\left(\mathrm{CF}_{3}\right) \mathrm{OSO}_{3} \mathrm{Na}\right.$ ]. 即使在烯烃的存在的条件下, $\alpha$-三氟甲 基芐溴在脱卤亚磺化条件下产生了自由基，也不与烯烃发生加成反应，而是直接生成亚磺酸盐，亚磺酸盐被空气氧化 成烷基硫酸盐.
\end{abstract}

关键词 三氟甲基; 自由基; 脱卤亚磺化; 烷基硫酸盐

\section{Synthesis of Alkyl Sulfate from $\alpha$-Trifluoromethylbenzylbromide- An Extension of Sulfinatodehalogenation}

\author{
$\mathrm{Fu}, \mathrm{Xiaolin}^{a}$ \\ Sun, Yan*,b \\ Zhao, Zhigang*,a \\ Guo, Yong ${ }^{b}$ \\ Chen, Qingyun ${ }^{b}$ \\ Nian, Baoyi ${ }^{c}$ \\ $\left({ }^{a}\right.$ College of Chemistry \& Environment Protection Engineering, Southwest Minzu University, Chengdu 610041) \\ $\left({ }^{b}\right.$ College of Chemistry \& Environment Protection Engineering, Shanghai Institute of Organic Chemistry, \\ Chinese Academy of Sciences, Shanghai 200032) \\ ( ${ }^{c}$ Sanming Institute of Fluorochemical Industry, Sanming University, Sanming 365004)
}

\begin{abstract}
The sulfinatodehalogenation reaction is a common method of introducing a fluoroalkyl group. In this paper, the reaction of $\alpha$-trifluoromethylbenzyl bromide under sulfinatodehalogenation conditions was investigated. It was found that the product was an sodium alkyl sulfate $\left(\mathrm{ArCH}\left(\mathrm{CF}_{3}\right) \mathrm{OSO}_{3} \mathrm{Na}\right)$ instead of an sodium alkyl sulfinate $\left(\mathrm{ArCH}\left(\mathrm{CF}_{3}\right) \mathrm{SO}_{2} \mathrm{Na}\right)$ which was normal produced. $\alpha$-Trifluoromethylbenzyl bromide did not react with the olefin after its generation of a radical intermediate under sulfinatodehalogenation conditions even though an olefin was presented. Instead, the reaction directly gave an alkyl sulfinate, and then oxidized by air to provide a product as an alkyl sulfate.
\end{abstract}

Keywords Trifiluoromethyl; free radical; sulfinatodehalogenation; alkyl sulfate

1989 年, 黄维垣小组报道了氟烷基碘化物的脱卤 亚磺化反应, 之后脱卤亚磺化反应得到了广泛的应用 ${ }^{[1]}$. 该反应具有反应条件温和, 操作简单, 底物普适性好, 反应效率高等特点. 氟烷基卤化物在脱卤亚磺化的条件 下产生氟烷基自由基，该自由基能够与烯烃、炔烃和芳 香化合物发生反应. 在没有不饱和化合物存在的条件 下，该氟烷基自由基会和体系中的二氧化硫阴离子自由
基结合，生成亚磺酸盐. 该亚磺酸盐可以和一些氧化剂 作用, 如氯气/溴水可将其转化成磺酰氯/溴, 碘将其氧 化后脱去二氧化硫生成全氟烷基碘. 在过氧化氢的作用 下，全氟烷基的亚磺酸盐会生成磺酸盐. 但是在已有的 文献中，我们没有发现亚磺酸盐进一步氧化生成烷基硫 酸盐的例子.

有机氟化学在有机化学的合成上发挥着巨大的作

\footnotetext{
* Corresponding authors. E-mail: sunyan@sioc.ac.cn; zzg63129@163.com

Received October 18, 2018; revised November 24, 2018; published online November 30, 2018.

Dedicated to Professor Qingyun Chen on the occasion of his 90th birthday.

Project supported by the National Natural Science Foundation of China (Nos. 21737004, 21672239, 21421002) and the Sanming Institute of Fluorochemical Industry (Nos. FCIT201704GR, FCIT201705GR, FCIT201701BR).

国家自然科学基金(Nos. 21737004, 21672239, 21421002)和三明市氟化工产业技术研究院(Nos. FCIT201704GR, FCIT201705GR, FCIT201701BR)资助 项目.
} 
用, 氟化学合成方法学和机理的研究为含氟化合物的合 成打下了坚实的基础 ${ }^{[2,3]}$. 三氟甲基叔碳化合物的合成 是一个颇具挑战性的课题. 合成这类化合物的方法有以 下几种: (一)芳基 $\alpha$-位的直接引入三氟甲基化的方法 ${ }^{[4]}$; （二） $\alpha$-三氟甲基醇的傅克芳基化反应的方法 ${ }^{[5]}$; (三)含三 氟甲基砌块的金属偶联的方法 ${ }^{[6]}$; (四)通过偕二氟烯烃 与过渡金属氟盐合成 $\alpha$-三氟甲基化合物的方法 ${ }^{[7]}$. 我们 探究了三氟乙基卤化物的脱卤亚磺化反应, 该反应能在 三氟甲基的 $\alpha$ 位上快速引入烷基基团 ${ }^{[8]}$. 并且我们小组、 Carreira 小组以及胡金波小组均研究了可见光促进的三 氟甲基 $\alpha$-位的自由基反应 ${ }^{[9]}$. 可见光促进的反应还可以 将 $\alpha$-三氟甲基澳代物转化成相应的自由基而与烯烃发 生加成反应 ${ }^{[10]}$. 我们设想从 $\alpha$-三氟甲基芐溴出发, 通过 自由基反应, 生成三氟甲基叔碳化合物. 经过一系列的 探究发现反应并没有生成预期的产物, 而是意外发现生 成物为单边酯化的硫酸盐, 即烷基硫酸盐(Scheme 1).
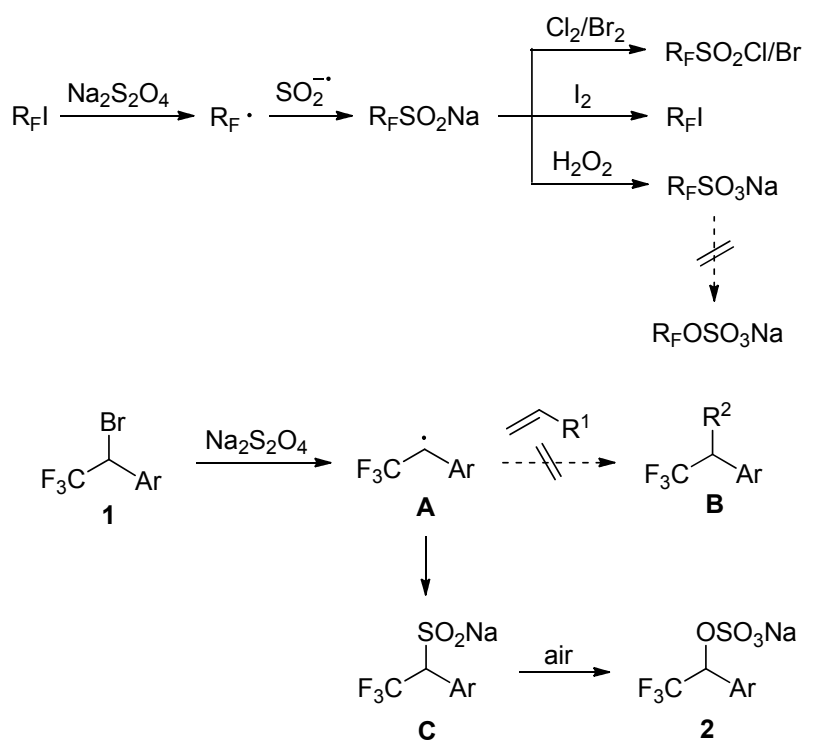

图式 1 亚磺酸盐的氧化及意外发现

Scheme 1 Oxidation and accidental discovery of sulfinate

烷基硫酸盐最常见的用途是作为表面活性剂, 如十 二烷基硫酸钠. 此类硫酸盐和单边酯化的磷酸盐都是生 命体中普遍存在的可溶性酯类物质, 因此很多药物分子 中都含有硫酸盐的结构 ${ }^{[11]}$. 合成单边酯化的硫酸盐常见 的方法有三类: 第一类为单边水解或氢化二烷基硫酸酯 的反应 ${ }^{[12]}$; 第二类为三氧化硫与醇类生成硫酸盐的反

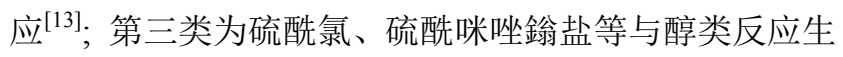
成硫酸盐的反应 ${ }^{[14]}$.

\section{1 结果与讨论}

在乙腈和水 $(V / V=2 / 1)$ 的混合溶液中, 在保险粉和 小苏打的共同作用下， $\alpha$-三氟甲基芐溴在 $50{ }^{\circ} \mathrm{C}$ 发生化
学反应(Scheme 2). 通过 ${ }^{19} \mathrm{~F}$ NMR 监测观察到, 原料 1a 的化学位移在 $\delta-70.4$, 反应 $1 \mathrm{~h}$ 后, 氟化学位移发生 明显变化, 在 $\delta-61.3$ 处出现双峰, 经计算这一步的氟 谱产率为 $99 \%$. 我们推测 $\delta-61.3$ 处的物质可能为亚磺 酸盐, 生成亚磺酸盐经历自由基 $\mathbf{A}$ 的过程(Scheme 1). 经查找已知文献对比可知含 $\alpha$-三氟甲基的醇类化合物 ${ }^{19} \mathrm{~F}$ NMR 化学位移在 $\delta-78{ }^{[15]}$; 含 $\alpha$-三氟甲基溴化物 ${ }^{19} \mathrm{~F}$ NMR 化学位移在 $\delta-70^{[15,16]}$; 三氟乙基亚磺酸钠 ${ }^{19} \mathrm{~F}$ NMR 化学位移在 $\delta-60^{[17,8 b]}$. 根据此类规律可以判 断中间体为亚磺酸盐. 并且, 即使在反应之初加入烯烃, 也没有观测到与烯烃的加成产物, 产物的化学位移没有 发生变化. 在后处理的过程中, 我们发现 $\delta-61.3$ 处的 双峰逐渐消失, 并在 $\delta-76.4$ 处出现双峰. 由以上现象, 我们推测发生了意料之外的反应.

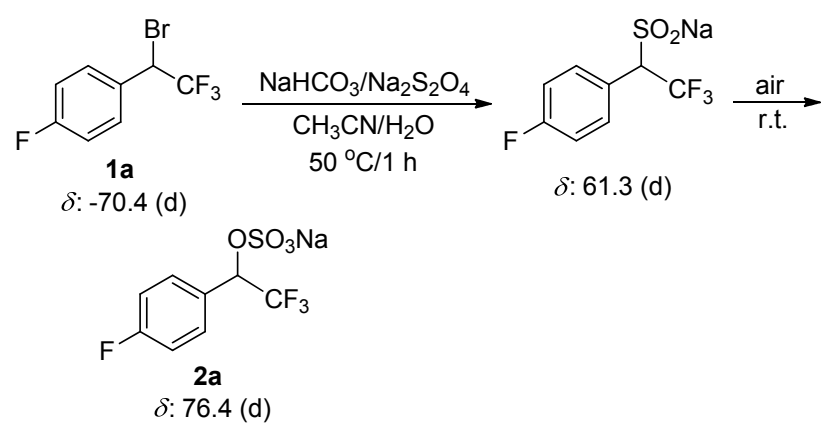

图式 $2 \alpha$-三氟甲基芐溴在脱卤亚磺化反应条件下的化学转 化

Scheme 2 Chemical conversion of $\alpha$-trifluoromethylbenzyl bromide under sulfinatodehalogenation

之后, 我们对这个化学位移在 $\delta-76.4$ 的物质进行 提纯, 发现该物质在水中的溶解度大于在乙醚中的溶解 度, 因此, 猜测该物质可能是一种盐. 我们用少量水将 反应体系进行溶解稀释, 并用乙醚进行多次萃取, 除去 水中的其他有机杂质，保证水层中的有机杂质被萃取完 全，对水层和乙醚层进行 ${ }^{19} \mathrm{~F}$ NMR 跟踪检测. 通过核磁 共振检测及上述处理, 判断水层含有无机盐和产物(即 $\delta$ -76.4 的物质). 因此我们用乙酸乙酯对水层进行多次 萃取, 旋蒸得到白色纯净固体, 根据 ${ }^{19} \mathrm{~F}$ NMR 检测, 该 固体的化学位移在 $\delta-76.4$, 即目标产物. 根据核磁共 振和 ESI 高低分辨质谱等检测手段, 我们判断生成的产 物是单边酯化的硫酸盐 $\mathbf{2 a}$, 分离产率为 $37 \%$, 该产率偏 低的原因是纯化过程中有损失. 因为该反应生成一些有 机杂质, 在上述除杂的过程中需要用乙醚进行多次萃 取, 一部分产物 2a 溶解到乙醚中造成损失, 导致目标物 产率偏低.

之后, 我们对亚磺酸盐转化成硫酸盐所需时间做了 观察. 在对亚磺酸盐进行提纯(约 $20 \mathrm{~min}$ )后, 经 ${ }^{19} \mathrm{~F}$ NMR 检测, 亚磺酸盐 $(\delta-61.3)$ 全部转化硫酸盐 $(\delta-$ 
76.4) (Scheme 2). 我们还在空气环境中直接进行了该反 应，结果发现反应 $1 \mathrm{~h}$ 后, $1 \mathbf{a}$ 也全部转化成 $\mathbf{2 a}$.

\section{1 底物普适性研究}

接下来, 我们对 $\alpha$-三氟甲基苠溴化合物与连二亚硫 酸钠反应的底物普适性进行了探究(表 1 所示). 从表 1 中可以看出，该第一步生成亚磺酸盐的反应，对于芳基 对位以及间位的吸电子基和给电子基类底物 $(\mathbf{1 a} \sim \mathbf{1 g}$, 1j)都得到中等到良好的产率(60\% 99\%); 对于芳基邻 位取代的底物(1h，1i)产率降低(55\%和 46\%); 同样该反 应也适合多取代结构, 对于 3,5-二甲氧基取代的 $\alpha$-三氟 甲基芳溴化合物(1k)也可以得到中等产率(52\%). 正如 前面所描述的，纯化烷基硫酸盐的过程有较大的损失， 因此, 分离得到的烷基硫酸盐的产率在 15\%到 40\%之 间.

最后我们对反应反应机理进行了研究(Scheme 3). 溴代物 1d 直接在乙腈的水溶液中反应, 部分溴代物会 转变为醇, 但是转化的时间较长, $24 \mathrm{~h}$ 只转化了不到 $10 \%$. 而且直接以醇作为原料在碳酸氢钠和保险粉的乙 腈溶液中进行反应, 结果发现醇并不参与反应, 反应体 系无变化. 由此说明反应不是溴代物转化成醇再生成硫 酸盐.<smiles>COc1ccc(C(O)C(Br)C(F)(F)F)cc1</smiles><smiles>COc1ccc(C(O)C(F)(F)F)cc1</smiles><smiles>O=S(=O)(O)C([Al])C(F)(F)F</smiles>

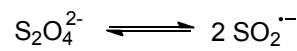
$+\mathrm{SO}_{2}^{--} \|-\mathrm{SO}_{2}^{--}$

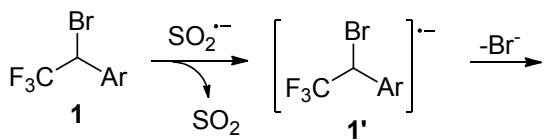
$\mathrm{F}_{3} \mathrm{C} \overbrace{\mathrm{Ar}}$ A $-\mathrm{O}_{2} \|+\mathrm{O}_{2}$

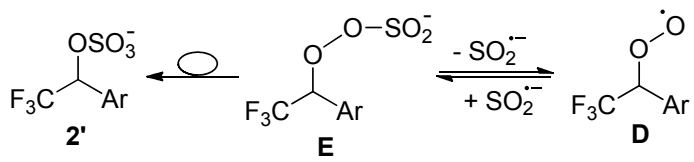

图式 3 对照实验和可能的氧化机理

Scheme 3 Comparative experiment and possible oxidation mechanism

我们推测的机理如 Scheme 3 所示. 溴代物 $\mathbf{1}$ 在脱卤 亚磺化条件会产生自由基 A. A 与二氧化硫阴离子自由 基结合生成烷基亚磺酸根 $\mathbf{C}^{\prime}$. 因为 $\mathbf{C}^{\prime}$ 中 $\mathrm{C}-\mathrm{S}$ 键结合很
弱，容易变回自由基 $\mathbf{A}$. 自由基 $\mathbf{A}$ 捕获 $\mathrm{O}_{2}$ 生成过氧自由 基 $\mathbf{D}$. 过氧自由基 $\mathbf{D}$ 与二氧化硫阴离子自由基结合生成 $\mathbf{E}, \mathbf{E}$ 经过重排最终得到稳定烷基硫酸根 $2^{\prime}$.

\section{2 结论}

三氟甲基苠溴在脱卤亚磺化的反应条件下，生成的 最终的产物是烷基硫酸盐, 这一反应是脱卤亚磺化反应 的延伸. 最终, 我们认为反应经过了亚磺酸盐的过程, 该亚磺酸盐会在空气中逐渐被氧化，最终生成硫酸盐.

\section{3 实验部分}

\section{1 仪器与试剂}

熔点通过 DSC 测定，温度未经校正; ${ }^{1} \mathrm{H}$ NMR 谱在 Agilent (400 MHz)上测定, 用 TMS 作为内标; ${ }^{19} \mathrm{~F} \mathrm{NMR}$ 谱在 Agilent (376 MHz) 上测定, 用 $\mathrm{CFCl}_{3}$ 和 $\mathrm{D}_{2} \mathrm{O}$ 作为内 标; ${ }^{13} \mathrm{C}$ NMR 谱在 Bruker AV-400 (100 MHz)上测定，用 $\mathrm{CDCl}_{3}$ 作为内标; HRMS (EI)在 SATURN 2000 型质谱仪 测定; MS (ESI)在 AGILENT1100 型质谱仪或 Shimadzu LCMS-2010EV 型质谱仪测定; HRMS (ESI)谱用 FTMS7 型质谱仪测定; 柱层析使用青岛海洋化工厂生产的硅 胶(200 300 目或 300 400 目). TLC 硅胶板为烟台化工 厂生产的 HSGF-254 型薄层层析预制板; 采用紫外灯显 色. 溶剂均以标准方法处理纯化.

\section{2 实验方法}

\section{$3.2 .1 \alpha$-三氟甲基苄溴的合成}

将三苯基膦( $\left.\mathrm{Ph}_{3} \mathrm{P}, 2 \mathrm{mmol}\right)$ 加入到 $10 \mathrm{~mL}$ Schlenk 管 中, 加入 $0.5 \mathrm{~mL}$ 甲苯溶液, 在 $50{ }^{\circ} \mathrm{C}$ 下搅拌溶解. 将 2,2,2-三氟甲基茮醇 $(1 \mathrm{mmol}$ )、四溴化碳 $(1 \mathrm{mmol}$ )和 $4 \mathrm{~mL}$ 甲苯溶液混合均匀, 将其加入到三苯基膦的甲苯溶液 中, $60{ }^{\circ} \mathrm{C}$ 下搅拌反应 $4 \mathrm{~h}$, 薄层色谱(TLC)检测. 反应完 全后过滤掉固体，用无水乙醚 $(5 \mathrm{~mL} \times 3)$ 稀释，用饱和碳 酸氢钠溶液洗涤 3 次, 合并有机相. 有机提取物用无水 $\mathrm{Na}_{2} \mathrm{SO}_{4}$ 干燥, 浓缩, 残留物用柱层析方法(乙酸乙酯和 石油醚体系或者正戊烷)分离纯化，得到目标产物 $\alpha$-三 氟甲基苄溴.

1-(1-溴-2,2,2-三氟乙基)-4-氟苯(1a) ${ }^{[18]}$ : 无色液体, 产率 79\%. ${ }^{1} \mathrm{H}$ NMR $\left(400 \mathrm{MHz}, \mathrm{CDCl}_{3}\right) \delta: 7.52 \sim 7.49(\mathrm{~m}$, $2 \mathrm{H}), 7.11 \sim 7.07(\mathrm{~m}, 2 \mathrm{H}), 5.12(\mathrm{q}, J=7.3 \mathrm{~Hz}, 1 \mathrm{H}) ;{ }^{19} \mathrm{~F}$ NMR (376 MHz, $\left.\mathrm{CDCl}_{3}\right) \delta:-70.8(\mathrm{~d}, J=7.1 \mathrm{~Hz}, 3 \mathrm{~F})$, -110.4 to $-110.5(\mathrm{~m}, 1 \mathrm{~F})$.

1,1,1-三氟-2-溴-2-苯乙烷 $(\mathbf{1 b})^{[19]}$ ：无色液体，产率 $76 \%$. ${ }^{1} \mathrm{H}$ NMR $\left(400 \mathrm{MHz}, \mathrm{CDCl}_{3}\right) \delta: 7.51 \sim 7.38(\mathrm{~m}, 5 \mathrm{H})$, 5.12 (q, $J=7.5 \mathrm{~Hz}, 1 \mathrm{H}) ;{ }^{19} \mathrm{~F}$ NMR $\left(376 \mathrm{MHz}, \mathrm{CDCl}_{3}\right) \delta$ : $-70.5(\mathrm{~d}, J=7.5 \mathrm{~Hz})$. 
表 $1 \alpha$-三氟甲基苄溴与连二亚硫酸钠的反应 ${ }^{a}$

Table 1 Reaction of $\alpha$-trifluoromethylbenzyl bromide with sodium dithionite

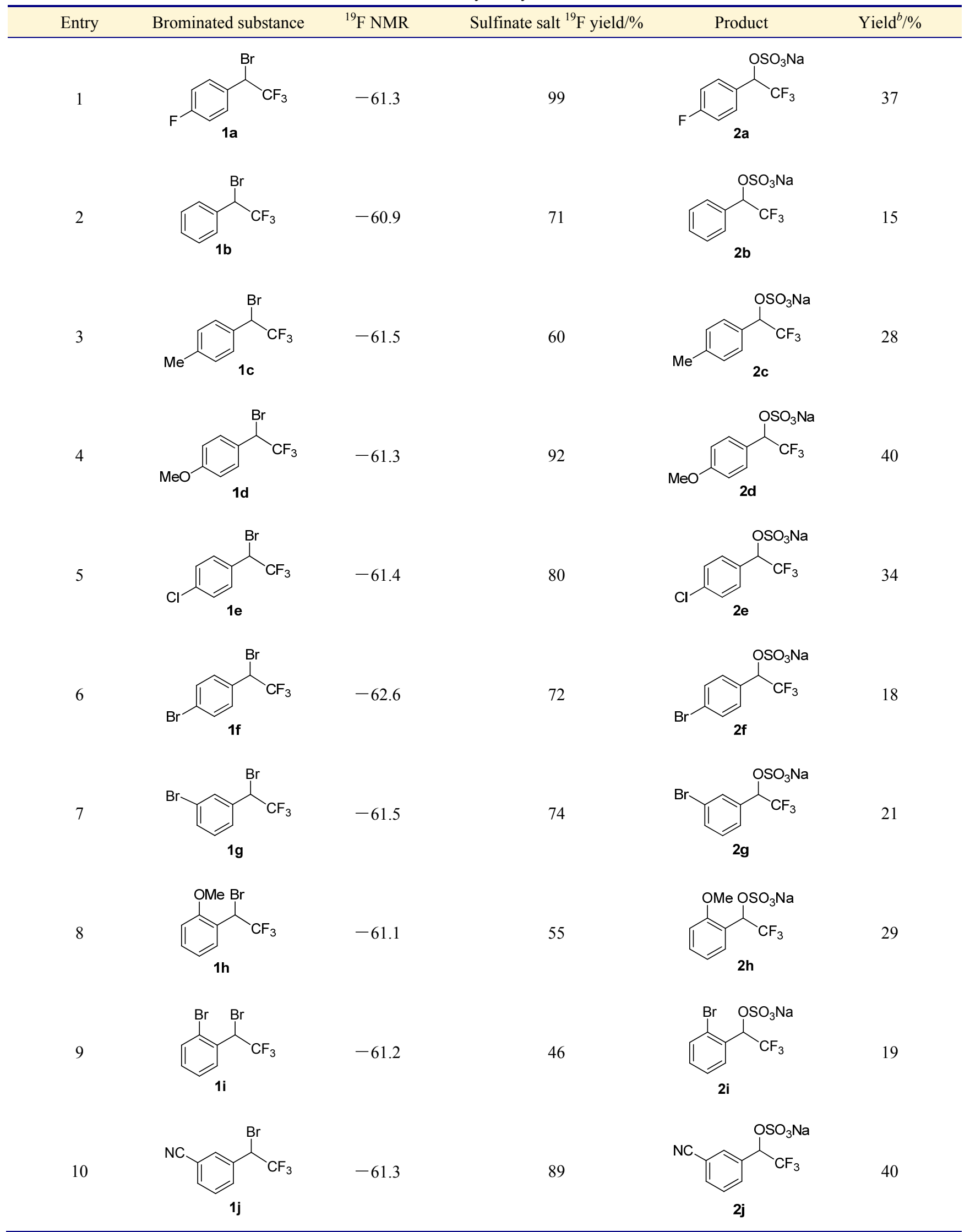

${ }^{a}$ Reaction condition: $1(0.5 \mathrm{mmol}), \mathrm{Na}_{2} \mathrm{~S}_{2} \mathrm{O}_{4}(0.75 \mathrm{mmol}), \mathrm{NaHCO}_{3}(0.75 \mathrm{mmol}), V\left(\mathrm{CH}_{3} \mathrm{CN}\right): V\left(\mathrm{H}_{2} \mathrm{O}\right)=2: 1(2.0 \mathrm{~mL})$ for $1 \mathrm{~h} .{ }^{b}$ Isolated yield. 
1-(1-溴-2,2,2-三氟乙基)-4-甲基苯(1c) ${ }^{[20]}$ : 无色液 体, 产率 60\%. ${ }^{1} \mathrm{H}$ NMR $\left(400 \mathrm{MHz}, \mathrm{CDCl}_{3}\right) \delta: 7.40(\mathrm{~d}, J=$ $8.0 \mathrm{~Hz}, 2 \mathrm{H}), 7.20$ (d, $J=7.4 \mathrm{~Hz}, 2 \mathrm{H}), 5.11$ (q, $J=7.5 \mathrm{~Hz}$, $1 \mathrm{H}), 2.38(\mathrm{~s}, 3 \mathrm{H}) ;{ }^{19} \mathrm{~F}$ NMR $\left(376 \mathrm{MHz}, \mathrm{CDCl}_{3}\right) \delta:-70.5$ (d, $J=7.5 \mathrm{~Hz})$.

1-(1-溴-2,2,2-三氟乙基)-4-甲氧基苯(1d $)^{[21]}$ : 无色液 体, 产率 60\%. ${ }^{1} \mathrm{H}$ NMR $\left(400 \mathrm{MHz}, \mathrm{CDCl}_{3}\right) \delta: 7.43(\mathrm{~d}, J=$ $8.0 \mathrm{~Hz}, 2 \mathrm{H}), 6.92 \sim 6.89(\mathrm{~m}, 2 \mathrm{H}), 5.11(\mathrm{q}, J=7.5 \mathrm{~Hz}, 1 \mathrm{H})$, $3.83(\mathrm{~s}, 3 \mathrm{H}) ;{ }^{19} \mathrm{~F}$ NMR $\left(376 \mathrm{MHz}, \mathrm{CDCl}_{3}\right) \delta:-70.7(\mathrm{~d}$, $J=7.5 \mathrm{~Hz})$.

1-(1-溴-2,2,2-三氟乙基)-4-氯苯(1e $)^{[22]: ~}$ 无色液体, 产率 80\%. ${ }^{1} \mathrm{H}$ NMR $\left(400 \mathrm{MHz}, \mathrm{CDCl}_{3}\right) \delta: 7.44(\mathrm{~d}, J=8.0$ $\mathrm{Hz}, 2 \mathrm{H}), 7.37$ (d, $J=8.0 \mathrm{~Hz}, 2 \mathrm{H}), 5.71(\mathrm{q}, J=6.7 \mathrm{~Hz}, 1 \mathrm{H})$; ${ }^{19} \mathrm{~F}$ NMR $\left(376 \mathrm{MHz}, \mathrm{CDCl}_{3}\right) \delta:-70.6(\mathrm{~d}, J=7.5 \mathrm{~Hz})$.

1-(1-澳-2,2,2-三氟乙基)-4-澳苯 (1f $)^{[23]}$ : 无色液体, 产率 75\%. ${ }^{1} \mathrm{H}$ NMR $\left(400 \mathrm{MHz}, \mathrm{CDCl}_{3}\right) \delta: 7.57 \sim 7.51(\mathrm{~m}$, $2 \mathrm{H}), 7.41 \sim 7.35(\mathrm{~m}, 2 \mathrm{H}), 5.08(\mathrm{q}, J=7.3 \mathrm{~Hz}, 1 \mathrm{H}) ;{ }^{19} \mathrm{~F}$ NMR $\left(376 \mathrm{MHz}, \mathrm{CDCl}_{3}\right) \delta$ : $-70.6(\mathrm{~d}, J=7.1 \mathrm{~Hz})$.

1-(1-溴-2,2,2-三氟乙基)-3-溴苯(19): 无色液体, 产 率 64\%. ${ }^{1} \mathrm{H}$ NMR $\left(400 \mathrm{MHz}, \mathrm{CDCl}_{3}\right) \delta: 7.66(\mathrm{~s}, 1 \mathrm{H})$, $7.56 \sim 7.53(\mathrm{~m}, 1 \mathrm{H}), 7.44(\mathrm{~d}, J=8 \mathrm{~Hz}, 1 \mathrm{H}), 7.28(\mathrm{t}, J=8$ $\mathrm{Hz}, 1 \mathrm{H}), 5.06(\mathrm{q}, J=7.3 \mathrm{~Hz}, 1 \mathrm{H}) ;{ }^{19} \mathrm{~F}$ NMR $(376 \mathrm{MHz}$, $\left.\mathrm{CDCl}_{3}\right) \delta:-70.4(\mathrm{~d}, J=7.1 \mathrm{~Hz})$.

1-(1-溴-2,2,2-三氟乙基)-2-甲氧基苯(1h): 无色液 体, 产率 75\%. ${ }^{1} \mathrm{H}$ NMR $\left(400 \mathrm{MHz}, \mathrm{CDCl}_{3}\right) \delta: 7.67$ (d, $J=$ $8.0 \mathrm{~Hz}, 1 \mathrm{H}), 7.39 \sim 7.35(\mathrm{~m}, 1 \mathrm{H}), 7.05 \sim 7.01(\mathrm{~m}, 1 \mathrm{H}), 6.91$ (d, $J=8.4 \mathrm{~Hz}, 1 \mathrm{H}), 5.90$ (q, $J=7.9 \mathrm{~Hz}, 1 \mathrm{H}), 3.89$ (s, 3H); ${ }^{19} \mathrm{~F} \mathrm{NMR}\left(376 \mathrm{MHz}, \mathrm{CDCl}_{3}\right) \delta:-70.0(\mathrm{~d}, J=7.5 \mathrm{~Hz}) ;{ }^{13} \mathrm{C}$ NMR $\left(100 \mathrm{MHz}, \mathrm{CDCl}_{3}\right) \delta: 156.5,131.2,130.3,123.8(\mathrm{q}$, $\left.J_{\mathrm{CF}}=276 \mathrm{~Hz}\right), 121.4,121.1,110.9,55.7,39.3\left(\mathrm{q}, J_{\mathrm{CF}}=35\right.$ $\mathrm{Hz}$ ). HRMS (ESI) calcd for $\mathrm{C}_{9} \mathrm{H}_{8} \mathrm{OF}_{3} \mathrm{Br} 267.9711$, found 267.9713.

1-溴-2-(1-溴-2,2,2-三氟乙基)苯(1i): 无色液体, 产 率 40\%. ${ }^{1} \mathrm{H}$ NMR $\left(400 \mathrm{MHz}, \mathrm{CDCl}_{3}\right) \delta: 7.79$ (d, $J=8.0$ $\mathrm{Hz}, 1 \mathrm{H}), 7.61 \sim 7.59(\mathrm{~m}, 1 \mathrm{H}), 7.42 \sim 7.38(\mathrm{~m}, 1 \mathrm{H}), 7.27 \sim$ $7.23(\mathrm{~m}, 1 \mathrm{H}), 5.85$ (q, $J=7.3 \mathrm{~Hz}, 1 \mathrm{H}) ;{ }^{19} \mathrm{~F}$ NMR $(376$ $\left.\mathrm{MHz}, \mathrm{CDCl}_{3}\right) \delta:-69.7(\mathrm{~d}, J=7.5 \mathrm{~Hz})$.

1-(1-溴-2,2,2-三氟乙基)-3-氧基苯(1j): 无色液体, 产率 40\%. ${ }^{1} \mathrm{H}$ NMR $\left(400 \mathrm{MHz}, \mathrm{CDCl}_{3}\right) \delta: 7.82(\mathrm{~s}, 1 \mathrm{H})$, $7.76(\mathrm{~d}, J=8.0 \mathrm{~Hz}, 1 \mathrm{H}), 7.72 \sim 7.70(\mathrm{~m}, 1 \mathrm{H}), 7.54(\mathrm{t}, J=$ $7.8 \mathrm{~Hz}, 1 \mathrm{H}), 5.14$ (q, $J=7.1 \mathrm{~Hz}, 1 \mathrm{H}) ;{ }^{19} \mathrm{~F}$ NMR $(376 \mathrm{MHz}$, $\left.\mathrm{CDCl}_{3}\right) \delta:-70.5(\mathrm{~d}, J=7.1 \mathrm{~Hz}) ;{ }^{13} \mathrm{C}$ NMR $(100 \mathrm{MHz}$, $\left.\mathrm{CDCl}_{3}\right) \delta: 134.4,133.5,133.4,132.8,129.9,123.0\left(\mathrm{q}, J_{\mathrm{CF}}=\right.$ $276 \mathrm{~Hz}), 117.7,113.4,45.4$ (q, $J_{\mathrm{CF}}=34 \mathrm{~Hz}$ ). HRMS (ESI) calcd for $\mathrm{C}_{9} \mathrm{H}_{5} \mathrm{NF}_{3} \mathrm{Br} 262.9557$, found 262.9561 .

\subsection{2 烷基硫酸盐的合成}

在 Schlenk 管中加入连二亚硫酸钠 $(0.75 \mathrm{mmol})$ 和碳 酸氢钠 $(0.75 \mathrm{mmol})$, 双排管严格充换氮气三次, 在氮气 保护下加入 $2 \mathrm{~mL}$ 乙腈水溶液 $\left[V\left(\mathrm{CH}_{3} \mathrm{CN}\right): V\left(\mathrm{H}_{2} \mathrm{O}\right)=\right.$ $2: 1]$. 于 Schlenk 管中加入 $\alpha$-三氟甲基芐溴 $(0.5 \mathrm{mmol})$, $50{ }^{\circ} \mathrm{C}$ 下搅拌反应 $1 \mathrm{~h}, \mathrm{TLC}$ 检测直到原料反应完全. ${ }^{19} \mathrm{~F}$ NMR 检测, 原料完全转化, 生成化学位移约在 $\delta-61$ 的双峰的产物. 停止反应后, 向反应混合物加入 $5 \mathrm{~mL}$ 水溶液, 乙醚 $(5 \mathrm{~mL})$ 萃取数次, 水层和乙醚层 ${ }^{19} \mathrm{~F} \mathrm{NMR}$ 检测, 直到水层仅剩余 $\delta-76$ 双峰的产物为止. 最后, 水层用乙酸乙酯 $(5 \mathrm{~mL} \times 3)$ 萃取, 合并有机相, 浓缩得到 白色固体为烷基硫酸盐.

2,2,2-三氟-1-(4-氟苯基)乙基硫酸钠(2a): 白色固体, 分离产率 37\%. m.p. $71.8{ }^{\circ} \mathrm{C} ;{ }^{1} \mathrm{H}$ NMR $\left(400 \mathrm{MHz}, \mathrm{D}_{2} \mathrm{O}\right)$ $\delta: 7.56 \sim 7.52(\mathrm{~m}, 2 \mathrm{H}), 7.17(\mathrm{t}, J=8.8 \mathrm{~Hz}, 2 \mathrm{H}), 5.71(\mathrm{q}$, $J=6.7 \mathrm{~Hz}, 1 \mathrm{H}) ;{ }^{19} \mathrm{~F}$ NMR $\left(376 \mathrm{MHz}, \mathrm{D}_{2} \mathrm{O}\right) \delta:-77.2(\mathrm{~d}$, $J=6.4 \mathrm{~Hz}, 3 \mathrm{~F}),-112.0$ to -112.1 (m, 1F). HRMS (ESI) calcd for $\mathrm{C}_{8} \mathrm{H}_{5} \mathrm{O}_{4} \mathrm{~F}_{4} \mathrm{SNa}\left[\mathrm{M}-\mathrm{Na}^{+}\right] 272.9850$, found 272.9853 .

2,2,2-三氟-1-苯基乙基硫酸钠(2b): 白色固体, 分离 产率 $15 \%$. m.p. $60.5{ }^{\circ} \mathrm{C} ;{ }^{1} \mathrm{H}$ NMR $\left(400 \mathrm{MHz}, \mathrm{D}_{2} \mathrm{O}\right) \delta$ : $7.54 \sim 7.45(\mathrm{~m}, 5 \mathrm{H}), 5.71(\mathrm{q}, J=6.7 \mathrm{~Hz}, 1 \mathrm{H}) ;{ }^{19} \mathrm{~F}$ NMR (376 MHz, $\left.\mathrm{D}_{2} \mathrm{O}\right) \delta$ : -77.0 (d, $J=6.8 \mathrm{~Hz}$ ). HRMS (ESI) calcd for $\mathrm{C}_{8} \mathrm{H}_{6} \mathrm{O}_{4} \mathrm{~F}_{3} \mathrm{SNa}\left[\mathrm{M}-\mathrm{Na}^{+}\right]$254.9944, found 254.9948 .

2,2,2-三氟-1-(对甲基苯基)乙基硫酸钠(2c)：白色固 体, 分离产率 28\%. m.p. $49.4{ }^{\circ} \mathrm{C} ;{ }^{1} \mathrm{H}$ NMR (400 MHz, $\left.\mathrm{D}_{2} \mathrm{O}\right) \delta: 7.40(\mathrm{~d}, J=8.0 \mathrm{~Hz}, 2 \mathrm{H}), 7.27(\mathrm{~d}, J=8.0 \mathrm{~Hz}, 2 \mathrm{H})$, 5.65 (q, $J=6.7 \mathrm{~Hz}, 1 \mathrm{H}), 2.30(\mathrm{~s}, 3 \mathrm{H}) ;{ }^{19} \mathrm{~F}$ NMR $(376 \mathrm{MHz}$, $\left.\mathrm{D}_{2} \mathrm{O}\right) \delta$ : $-77.1(\mathrm{~d}, J=5.3 \mathrm{~Hz}$ ). HRMS (ESI) calcd for $\mathrm{C}_{9} \mathrm{H}_{8} \mathrm{O}_{4} \mathrm{~F}_{3} \mathrm{SNa}\left[\mathrm{M}-\mathrm{Na}^{+}\right]$269.0101, found 269.0099.

2,2,2-三氟-1-(4-甲氧基苯基)乙基硫酸钠 (2d): 白色 固体, 分离产率 $40 \%$. m.p. $46.6{ }^{\circ} \mathrm{C} ;{ }^{1} \mathrm{H}$ NMR $(400 \mathrm{MHz}$, $\left.\mathrm{D}_{2} \mathrm{O}\right) \delta: 7.50(\mathrm{~d}, J=8.8 \mathrm{~Hz}, 2 \mathrm{H}), 7.05(\mathrm{~d}, J=8.8 \mathrm{~Hz}, 2 \mathrm{H})$, $5.68(\mathrm{q}, J=6.5 \mathrm{~Hz}, 1 \mathrm{H}), 3.83(\mathrm{~s}, 3 \mathrm{H}) ;{ }^{19} \mathrm{~F}$ NMR $(376 \mathrm{MHz}$, $\left.\mathrm{D}_{2} \mathrm{O}\right) \delta$ : $-77.1(\mathrm{~d}, J=6.8 \mathrm{~Hz}$ ). HRMS (ESI) calcd for $\mathrm{C}_{9} \mathrm{H}_{8} \mathrm{O}_{5} \mathrm{~F}_{3} \mathrm{SNa}\left[\mathrm{M}-\mathrm{Na}^{+}\right]$285.0050, found 285.0048.

1-(4-氯苯基)-2,2,2-三氟乙基硫酸钠 (2e): 白色固体, 分离产率 34\%. m.p. $71.0{ }^{\circ} \mathrm{C} ;{ }^{1} \mathrm{H}$ NMR $\left(400 \mathrm{MHz}, \mathrm{D}_{2} \mathrm{O}\right)$ $\delta: 7.98(\mathrm{~s}, 1 \mathrm{H}), 7.86(\mathrm{t}, J=8.6 \mathrm{~Hz}, 2 \mathrm{H}), 7.64(\mathrm{t}, J=8.0 \mathrm{~Hz}$, $1 \mathrm{H}), 5.85(\mathrm{q}, J=6.5 \mathrm{~Hz}, 1 \mathrm{H}) ;{ }^{19} \mathrm{~F}$ NMR $\left(376 \mathrm{MHz}, \mathrm{D}_{2} \mathrm{O}\right) \delta$ : -76.9 (d, $J=6.4 \mathrm{~Hz}$ ). HRMS (ESI) calcd for $\mathrm{C}_{8} \mathrm{H}_{5} \mathrm{O}_{4^{-}}$ $\mathrm{ClF}_{3} \mathrm{SNa}\left[\mathrm{M}-\mathrm{Na}^{+}\right]$288.9549, found 288.9548 .

1-(4-溴苯基)-2,2,2-三氟乙基硫酸钠(2f): 白色固体, 产率 $18 \%$. m.p. $65.9{ }^{\circ} \mathrm{C} ;{ }^{1} \mathrm{H}$ NMR $\left(400 \mathrm{MHz}, \mathrm{D}_{2} \mathrm{O}\right) \delta$ : 
$7.69(\mathrm{t}, J=8.8 \mathrm{~Hz}, 2 \mathrm{H}), 7.51 \sim 7.35(\mathrm{~m}, 2 \mathrm{H}), 6.21(\mathrm{q}, J=$ $6.4 \mathrm{~Hz}, 1 \mathrm{H}) ;{ }^{19} \mathrm{~F}$ NMR $\left(376 \mathrm{MHz}, \mathrm{D}_{2} \mathrm{O}\right) \delta:-76.6(\mathrm{~d}, J=$ $6.4 \mathrm{~Hz}$ ). HRMS (ESI) calcd for $\mathrm{C}_{8} \mathrm{H}_{5} \mathrm{O}_{4} \mathrm{~F}_{3} \mathrm{SNa}\left[\mathrm{M}-\mathrm{Na}^{+}\right]$ 332.9049, found 332.9053 .

1-(3-溴苯基)-2,2,2-三氟乙基硫酸钠(2g): 白色固体, 分离产率 45\%. m.p. $110.5{ }^{\circ} \mathrm{C} ;{ }^{1} \mathrm{H}$ NMR (400 MHz, $\left.\mathrm{D}_{2} \mathrm{O}\right)$ $\delta: 7.77(\mathrm{~s}, 1 \mathrm{H}), 7.65(\mathrm{~d}, J=8.0 \mathrm{~Hz}, 1 \mathrm{H}), 7.53(\mathrm{~d}, J=8.0$ $\mathrm{Hz}, 1 \mathrm{H}), 7.40 \sim 7.33(\mathrm{~m}, 1 \mathrm{H}), 5.74(\mathrm{q}, J=6.5 \mathrm{~Hz}, 1 \mathrm{H}) ;{ }^{19} \mathrm{~F}$ NMR $\left(376 \mathrm{MHz}, \mathrm{D}_{2} \mathrm{O}\right) \delta$ : $-76.9(\mathrm{~d}, J=6.4 \mathrm{~Hz})$. HRMS (ESI) calcd for $\mathrm{C}_{8} \mathrm{H}_{5} \mathrm{O}_{4} \mathrm{BrF}_{3} \mathrm{SNa}\left[\mathrm{M}-\mathrm{Na}^{+}\right] 332.9049$, found 332.9050 .

2,2,2-三氟-1-(2-甲氧基苯基)乙基硫酸钠 $(\mathbf{2 h})$ : 白色 固体, 分离产率 $29 \%$. m.p. $78.5{ }^{\circ} \mathrm{C} ;{ }^{1} \mathrm{H}$ NMR (400 MHz, $\left.\mathrm{D}_{2} \mathrm{O}\right) \delta: 7.55(\mathrm{~d}, J=8.0 \mathrm{~Hz}, 1 \mathrm{H}), 7.48 \sim 7.38(\mathrm{~m}, 1 \mathrm{H})$, $7.11 \sim 7.04(\mathrm{~m}, 2 \mathrm{H}), 6.17$ (q, $J=6.5 \mathrm{~Hz}, 1 \mathrm{H}), 3.84(\mathrm{~s}, 3 \mathrm{H})$; ${ }^{19} \mathrm{~F}$ NMR $\left(376 \mathrm{MHz}, \mathrm{D}_{2} \mathrm{O}\right) \delta:-77.1(\mathrm{~d}, J=6.8 \mathrm{~Hz})$. HRMS (ESI) calcd for $\mathrm{C}_{9} \mathrm{H}_{8} \mathrm{O}_{5} \mathrm{~F}_{3} \mathrm{SNa}\left[\mathrm{M}-\mathrm{Na}^{+}\right] 285.0050$, found 285.0053 .

1-(2-溴苯基)-2,2,2-三氟乙基硫酸钠(2i): 白色固体, 分离产率 19\%. m.p. $57.7{ }^{\circ} \mathrm{C} ;{ }^{1} \mathrm{H}$ NMR (400 MHz, $\left.\mathrm{D}_{2} \mathrm{O}\right)$ $\delta$ : 7.69 (t, $J=8.0 \mathrm{~Hz}, 2 \mathrm{H}), 7.49$ (t, $J=5.3 \mathrm{~Hz}, 1 \mathrm{H}), 7.39 \sim$ $7.35(\mathrm{~m}, 1 \mathrm{H}), 6.21$ (q, $J=6.7 \mathrm{~Hz}, 1 \mathrm{H}) ;{ }^{19} \mathrm{~F}$ NMR $(376$ $\left.\mathrm{MHz}, \mathrm{D}_{2} \mathrm{O}\right) \delta$ : $-76.6(\mathrm{~d}, J=6.4 \mathrm{~Hz}$ ). HRMS (ESI) calcd for $\mathrm{C}_{8} \mathrm{H}_{5} \mathrm{O}_{4} \mathrm{BrF}_{3} \mathrm{SNa}\left[\mathrm{M}-\mathrm{Na}^{+}\right]$332.9049, found 332.9053.

1-(3-氰基苯基)-2,2,2-三氟乙基硫酸钠 $(2 \mathbf{j})$ : 白色固 体, 分离产率 $40 \%$. m.p. $60.2{ }^{\circ} \mathrm{C} ;{ }^{1} \mathrm{H}$ NMR $(400 \mathrm{MHz}$, $\left.\mathrm{D}_{2} \mathrm{O}\right) \delta: 7.98(\mathrm{~s}, 1 \mathrm{H}), 7.86(\mathrm{t}, J=8.6 \mathrm{~Hz}, 2 \mathrm{H}), 7.64(\mathrm{t}, J=$ $8.0 \mathrm{~Hz}, 1 \mathrm{H}), 5.85$ (q, $J=6.5 \mathrm{~Hz}, 1 \mathrm{H}) ;{ }^{19} \mathrm{~F}$ NMR $(376 \mathrm{MHz}$, $\left.\mathrm{D}_{2} \mathrm{O}\right) \delta:-76.9(\mathrm{~d}, J=6.4 \mathrm{~Hz})$. HRMS (ESI) calcd for $\mathrm{C}_{9} \mathrm{H}_{5} \mathrm{O}_{4} \mathrm{NF}_{3} \mathrm{SNa}\left[\mathrm{M}-\mathrm{Na}^{+}\right] 279.9897$, found 279.9891 .

\section{辅助材料(Supporting Information) 提供了化合物 ${ }^{1} \mathrm{H}$} $\mathrm{NMR},{ }^{19} \mathrm{~F} \mathrm{NMR},{ }^{13} \mathrm{C} \mathrm{NMR}$ 核磁谱图以及高分辨质谱数 据. 这些材料可以免费从本刊网站(http://sioc-journal. $\mathrm{cn} /)$ 上下载.

\section{References}

[1] (a) Huang, W.-Y. Organofluorine Chemistry in China, Shanghai Scientific and Technical Publishers, Shanghai, 1996 (in Chinese). (黄维垣, 中国有机氟化学研究, 上海科学技术出版社, 上海, 1996.)

(b) Zhang, C.-P.; Guo, Y.; Xiao, J.-C.; Gu, Y.-C. Chem. Soc. Rev. 2012, 41, 4536 .

(c) Huang, B.-N.; Huang, W.-Y.; Hu, C.-M. Acta Chim. Sinica 1981, 39, 481 (in Chinese).

(黄炳南, 黄维垣, 胡昌明, 化学学报, 1981, 39, 481.)

(d) Huang, W.-Y.; Huang, B.-N.; Hu, C.-M. J. Fluorine Chem. 1983, 23, 193 (in Chinese).

[2] (a) Qing, F.-L.; Qiu, X.-L. Organofluorine Chemistry, Science Press, Beijing, 2007 (in Chinese).
(卿凤翎，邱小龙，有机氟化学，科学出版社，北京, 2007.)

(b) Prakash, K. Modern Fluoroorganic Chemistry. Synthesis, Reactivity, Applications, 2nd ed., Wiley-VCH, Weinheim, 2013.

(c) Uneyama, K. Organofluorine Chemistry, Blackwell, Oxford, UK, 2006.

(d) Valentine, G. N.; Vasiliy, M. M.; Aleksey, V. S. Chem. Rev. 2015, 115, 973.

(e) Zhang, C.-P.; Chen, Q.-Y.; Guo, Y.; Xiao, J.-C.; Gu, Y.-C. Coord. Chem. Rev. 2014, 261, 28.

(f) Guo, Y.; Huang, M.-W.; Fu, X.-L.; Liu, C.; Chen, Q.-Y.; Zhao, Z.-G. Chin. Chem. Lett. 2017, 28, 719.

(g) Li, G.-M.; Sun, D.-Q. Chin. J. Org. Chem. 2016, 36, 1715 (in Chinese).

(李恭铭, 孙德群，有机化学, 2016, 36, 1715.)

(h) He, J.-Q.; Lou, S.-J.; Xu, D.-Q. Acta Chim. Sinica 2016, 36, 1218 (in Chinese).

(何将旗, 娄绍杰, 许丹倩, 化学学报, 2016, 36, 1218.)

(i) Xu, Y.-Y.; Qian, A.-R.; Cao, X.-F.; Ling, C.-Y.; Cao, Y.-B.; Wang, R.-L.; Li, Y.-S.; Yang, Y.-S. Chin. Chem. Lett. 2016, 27, 703. (j) Fu, L.-N.; Leng, B.; Li, Y.-S.; Gao, X.-K. Chin. Chem. Lett. 2016, 27, 1319.

[3] (a) Han, E.; Guo, Y.; Chen, Q.-Y.; Chin. J. Org. Chem. 2017, 37, 1714 (in Chinese).

(韩恩健，郭 勇，陈庆云，有机化学, 2017, 37, 1714.)

(b) Yang, B.; Xu, X.-H.; Qing, F.-L. Chin. J. Chem. 2016, 34, 465.

(c) Zhang, Z.; Yu, W.; Zhou, Q.; Li, T.; Zhang, Y.; Wang, J. Chin. J. Chem. 2016, 34, 473.

(d) Zhang, B.; Zhang, X.-G. Chin. J. Chem. 2016, 34, 477.

(e) Geng, Q.; Xiao, X.; He, G.-R.; Yao, S.-M.; Liang, G.-X. Chin. Chem. Lett. 2016, 27, 1009.

(f) Zhang, B.-H.; Kong, J.-J.; Huang, Y.; Lou, Y.-G.; Li, X.-F.; He, C.-Y. Chin. Chem. Lett. 2017, 28, 1751.

(g) Liu, Y.-L.; Yin, X.-P.; Zhou, J. Chin. J. Chem. 2018, 36, 321.

[4] (a) Miyake, Y.; Ota, S.-I.; Shibata, M.; Nakajima, K.; Nishibayashi, Y. Org. Biomol. Chem. 2014, 12, 5594.

(b) Egami, H.; Ide, T.; Kawato, Y.; Hamashima, Y. Chem. Commun. 2015, 51, 16675 .

(c) Zhu, L.; Liu, S.; Douglas, J. T.; Altman, R. A. Chem. Eur. J. 2013, 19, 12800

(d) Kuninobu, Y.; Nagase, M.; Kanai, M. Angew. Chem., Int. Ed. 2015, 54, 10263 .

(e) Wang, X.; Song, S.; Jiao, N. Chin. J. Chem. 2018, 36, 213 216.

[5] (a) Vuković, V. D.; Richmond, E.; Wolf, E.; Moran, J. Angew. Chem., Int. Ed. 2017, 56, 3085.

(b) Prakash, G. K. S.; Paknia, F.; Thomas M.; Mathew, T.; Mlostoń, G.; Olah, G. A. Org. Lett. 2011, 13, 4128.

[6] (a) Liang, Y.; Fu, G. C. J. Am. Chem. Soc. 2015, 137, 9523.

(b) Liang, Y.; Fu, G. C. Angew. Chem., Int. Ed. 2015, 54, 9047.

(c) Ryu, D.; Primer, D. N.; Tellis, J. C.; Molander, G. A. Chem. Eur. J. 2016, 22, 120

(d) Li, X.; Feng, Z.; Jiang, Z.-X.; Zhang, X. Org. Lett. 2015, 17, 5570 .

[7] (a) Gao, B.; Zhao, Y.; Hu, J. Angew. Chem., Int. Ed. 2015, 54, 638. (b) Tang, H.-J.; Zhang, Y.-F.; Jiang, Y.-W.; Feng, C. Org. Lett. 2018 , 20, 5190.

(c) Tian, P.; Wang, C.-Q.; Cai, S.-H.; Song, S.; Ye, L.; Feng, C.; Loh, T.-P. J. Am. Chem. Soc. 2016, 138, 15869.

(d) Tang, H.-J.; Lin, L.-Z.; Feng, C.; Loh T.-P. Angew. Chem., Int. Ed. 2017, 56, 9872.

[8] (a) Long, Z.-Y., Chen, Q.-Y. Tetrahedron Lett. 1998, 39, 8487. (b) Long, Z.-Y.; Chen, Q.-Y. J. Org. Chem. 1999, 64, 4775.

[9] (a) Li, L.; Huang, M.; Liu, C.; Xiao, J.-C.; Chen, Q.-Y.; Guo, Y.; Zhao, Z.-G. Org. Lett. 2015, 17, 4714.

(b) Huang M.; Li, L.; Zhao, Z.-G.; Chen, Q.-Y.; Guo, Y. Synth. Catal. 2015, 47, 3891 .

(c) Kreis, L. M.; Krautwald, S.; Pfeiffer, N.; Martin, R. E.; Carreira, E. M. Org. Lett. 2013, 15, 1634.

(d) Rong, J.; Ni, C.; Wang, Y.; Kuang, C.; Gu, Y.; Hu, J. Acta Chim. 
Sinica 2017, 75, 105.

[10] Huan, F., Chen, Q.-Y., Guo, Y. J. Org. Chem. 2016, 81, 7051.

[11] (a) Kudova, E.; Chodounska, H.; Slavikova, B.; Budesinsky, M.; Nekardova, M.; Vyklicky, V.; Krausova, B.; Svehla, P.; Vyklicky, L. J. Med. Chem. 2015, 58, 5950 .

(b) Burlingham, B. T.; Pratt L. M.; Davidson, E. R.; Shiner, V. J.; Jr.; Fong, J.; Widlanski, T. S. J. Am. Chem. Soc. 2003, 125, 13036.

[12] Kamiyama, H.; Kubo, Y.; Sato, H.; Yamamoto, N.; Fukuda, T.; Ishibashi, F.; Iwao, M. Bioorg. Med. Chem. 2011, 19, 7541.

[13] (a) Stastnaa, E.; Chodounskaa, H.; Pouzara, V.; Kaprasa, V.; Borovskaa, J.; Caisc, O.; Jr, L. V. Steroids 2009, 74, 256.

(b) Nishimura, Y.; Shudo, H.; Seto, H.; Hoshino, Y.; Miura, Y. Bioorg. Med. Chem. Lett. 2013, 23, 6390.

[14] (a) Nie, L.; S. Yao; Dong, B.; Li, X.-1.; Song, H. J. Mol. Liq. 2017, 240, 152.

(b) Desoky, A. Y.; Hendel, J.; Ingram L.; Taylor, S. D. Tetrahedron 2011, 67, 1281

(c) Beichel, W.; Panzer, J. M. U.; Hätty, J. Ye, X.; Himmel, D.;
Krossing, I. Angew. Chem., Int. Ed. 2014, 53, 6637.

[15] Dolbier, W. R.; Jr. Guide to Fluorine NMR for Organic Chemists, John Wiley \& Sons, Inc., New Jersey, 2009, p. 143.

[16] (a) Brunelle, J. A.; Letendre, L. J.; Weltin, E. E.; Brown, J. H.; Bushweller, C. H. J. Phys. Chem. 1992, 96, 9225.

(b) Tordeux, M.; Wakselman, C.; Jarjayes, O.; Béguin, C. G. Magn. Reson. Chem. 2001, 39, 301.

[17] Wang, R.; Jiang, L.; Yi, W. J. Org. Chem. 2018, 83, 7789.

[18] Dneprovskii, A. S.; Eliseenkov, E. V.; Mil'tsov, S. A. J. Org. Chem. 1982, 2, 365

[19] Yusuke, Y.; Shoji, H.; Hisanori, S. Tetrahedron 2010, 2, 473.

[20] Okano, T ; Fumoto, M ; Kusukawa, T ; Fujita, M. J. Fluorine Chem. 2002, 9, 91.

[21] Andreea, A. O.; Daweon, R.; Ioana, A.; Gary A, M. Angew. Chem., Int. Ed. 2013, 51, 13901

[22] Lo, W. C.; Hunter, J. E.; Watson, G. B.; Patny, A.; Iyer, P. S.; Boruwa, J. US 9211280, 2015

[23] Sun, F.; Zhan, Y. CN 108341814, 2018

(Li, L.; Fan, Y.) 